\title{
Attempted Depletion of Passenger Leukocytes by Irradiation in Pigs
}

\section{Hao-Chih Tai, ${ }^{1,2}$ Xiaocheng Zhu, ${ }^{1}$ Yih Jyh Lin, ${ }^{1}$ Hidetaka Hara, ${ }^{1}$ Mohamed Ezzelarab, ${ }^{1}$ Michael Epperley, ${ }^{3}$ Mubina A. Quader, ${ }^{3}$ and David K. C. Cooper ${ }^{1}$}

\author{
${ }^{1}$ Thomas E. Starzl Transplantation Institute, University of Pittsburgh Medical Center, Pittsburgh, PA 15261, USA \\ ${ }^{2}$ Department of Surgery, National Taiwan University Hospital and College of Medicine, Taipei 100, Taiwan \\ ${ }^{3}$ Department of Radiation Oncology, University of Pittsburgh Medical Center, Pittsburgh, PA 15261, USA
}

Correspondence should be addressed to David K. C. Cooper, cooperdk@upmc.edu

Received 21 July 2011; Revised 1 September 2011; Accepted 18 September 2011

Academic Editor: Kazuhiko Yamada

Copyright (c) 2011 Hao-Chih Tai et al. This is an open access article distributed under the Creative Commons Attribution License, which permits unrestricted use, distribution, and reproduction in any medium, provided the original work is properly cited.

\begin{abstract}
Allograft/xenograft rejection is associated with "passenger leukocyte" migration from the organ into recipient lymph nodes. In Study 1, we attempted to deplete leukocytes from potential kidney "donor" pigs, using two regimens of total body irradiation. A dose of 700 cGy was administered, followed by either 800 cGy ("low-dose") or 1,300 cGy ("high dose") with the kidneys shielded. Neither regimen was entirely successful in depleting all leukocytes, although remaining $\mathrm{T}$ and 8 cell numbers were negligible. Study 2 was aimed at providing an indication of whether near-complete depletion of leukocytes had any major impact on kidney allograft survival. In non-immunosuppressed recipient pigs, survival of a kidney from a donor that received high-dose irradiation was compared with that of a kidney taken from a non-irradiated donor. Kidney graft survival was 9 and 7 days, respectively, suggesting that depletion had little impact on graft survival. The lack of effect may have been related to (i) inadequate depletion of passenger leukocytes, thus not preventing a direct $\mathrm{T}$ cell response, (ii) the presence of dead or dying leukocytes (antigens), thus not preventing an indirect $\mathrm{T}$ cell response, or (iii) constitutive expression of MHC class II and B7 molecules on the porcine vascular endothelium, activating recipient $\mathrm{T}$ cells.
\end{abstract}

\section{Introduction}

Passenger leukocytes (contained within a transplanted organ and which migrate from the graft into the lymphoid tissues of the recipient) have complex dual roles in determining the outcome after transplantation (Tx). Donor-derived passenger leukocytes can initiate graft rejection in some settings, whereas in others they contribute to graft acceptance [1-3]. In a small animal model, donor pretreatment with sublethal total body irradiation (TBI) or antilymphocyte globulin resulted in significant prolongation of heart allograft survival [4]. In animal models of intestinal $\mathrm{Tx}$, passenger leukocytes in the intestine have a lineage profile that predisposes to graft-versus-host disease, which can be eliminated by ex vivo irradiation (10 Gy) of mature lymphoid elements from the bowel allografts [5]. In contrast, lethal TBI of the donor resulted in loss of ability to achieve tolerance in a pig kidney alloTx model [6].
The absolute number of MHC class $\mathrm{II}^{+}$dendritic cells present in a donor organ is a poor predictor of graft outcome, and survival of solid organ allografts is more closely related to the density of the donor dendritic cell network within the graft [7]. Prior irradiation of the potential organ donor leads to killing of the passenger leukocytes. Complete depletion of passenger leukocytes would likely prevent the direct T-cell response, though not the indirect, but might impact subsequent kidney graft survival.

The primary aim of the present study, therefore, was to determine whether a sufficient dose of irradiation could be administered to the "donor" pig to kill the leukocytes without seriously damaging the kidney (to be transplanted). The secondary aim was to determine whether complete or nearcomplete depletion of passenger leukocytes delayed rejection after kidney Tx.

Total body irradiation of the donor of $700 \mathrm{cGy}$ with a further $800 \mathrm{cGy}$ or $1,300 \mathrm{cGy}$ with the kidneys shielded proved 
insufficient to deplete all leukocytes. However, the function of the irradiated kidney and any possible protection from rejection was tested by Tx into a recipient pig without immunosuppressive therapy.

\section{Methods}

2.1. Animals. Outbred large white landrace pigs (Wally Whippo, Enon Valley, PA), aged 4-10 months, weighing 20$50 \mathrm{~kg}$, were used to assess two irradiation regimens $(n=3$ in each group) and as sources of kidneys $(n=3)$ for Tx into recipients $(n=3)$. All animal care and procedures were in accordance with the Principles of Laboratory Animal Care formulated by the National Society for Medical Research and the Guide for the Care and Use of Laboratory Animals prepared by the Institute of Laboratory Animal Resources and published by the National Institutes of Health (NIH publication number 86-23, revised 1985).

2.2. Irradiation. Pigs were sedated with ketamine $(7.5 \mathrm{mg} / \mathrm{kg}$ IV) and received TBI of $700 \mathrm{cGy}$ (350 cGy to each side) and then, on the same occasion, received a further 800 or 1300 cGy ( 400 or 650 cGy to each side) with both kidneys shielded. The dosages were determined from a review of the literature $[6,8-10]$. The pigs were irradiated using a $6 \mathrm{MV}$ photon beam from a Varian CLINAC 600C linear accelerator (Varian Medical System, Palo Alto, CA). TBI was performed by placing the pigs on the floor at an extended distance of $230 \mathrm{~cm}$ from the source and using a collimated beam of $40 \times 40 \mathrm{~cm}^{2}$, with the beam pointing downward towards the floor. This approach accommodated the entire body of the pig in the irradiation area. The pigs were radiated sequentially in right and left lateral decubitus positions with the longitudinal axis of the pigs placed along the horizontal axis of the radiation field. The prescribed dose was delivered to the midbody using equally weighted parallel opposed lateral fields. A dose rate of $250 \mathrm{MU} / \mathrm{min}$ was used during irradiation. Dose rates at different depths were measured under irradiation conditions to calculate radiation time and monitor units. The thickness of the pigs at midbody varied from 15$21 \mathrm{~cm}$. To accomplish shielding of the kidneys, the positions of the kidneys were identified using ultrasound imaging and their sites indicated by marking the skin. Both kidneys were protected from the second dose of irradiation by lead shields.

\subsection{Surgical Procedures}

2.3.1. Insertion of Intravenous Catheters. In all pigs, the right internal and external jugular veins were cannulated with silastic catheters, and the cannulae tunneled under the skin to the back, where they were brought out through the skin. These allowed access for drug administration and blood withdrawal for monitoring of renal function, tacrolimus levels (where indicated), and in vitro assays throughout the course of the experiment.

2.3.2. Kidney Transplantation. Life-supporting kidney $\mathrm{Tx}$ was carried out to leave no doubt that the survival of the pig depended on the function of the transplanted kidney. The right kidney of the donor pig was perfused with $2 \mathrm{~L}$ cold $\left(4^{\circ} \mathrm{C}\right)$ University of Wisconsin solution before nephrectomy. The recipient pig underwent right nephrectomy and kidney Tx. End-to-side anastomoses were carried out between the donor renal artery and recipient aorta and the donor renal vein and recipient inferior vena cava. The donor ureter was implanted into the recipient bladder. A double-J stent was placed from the pelvis of the kidney graft to the recipient bladder to maintain patency of the ureteric anastomosis. When the kidney graft had shown good urine output for $>30 \mathrm{~min}$, the native contralateral ureter was doubly ligated. Graft function was monitored daily by serum creatinine. A serum creatinine of $>884 \mu \mathrm{mol} / \mathrm{L}(>10 \mathrm{mg} / \mathrm{dL})$ indicated failure of the kidney.

2.3.3. Immunosuppressive Therapy. In one recipient pig, during the operative procedure (Day 0 ) immunosuppression was initiated with tacrolimus (Prograf, Astellas Pharma US, Deerfield, IL) at $0.1 \mathrm{mg} / \mathrm{kg} /$ day by i.v. infusion. Thereafter, tacrolimus was given i.m. at a dose of approximately $0.05 \mathrm{mg} / \mathrm{kg} \times 2$ daily, the dose being adjusted to maintain a $12 \mathrm{~h}$ blood trough level of $10-15 \mathrm{ng} / \mathrm{mL}$. Blood levels of tacrolimus were monitored daily to ensure the dosage was optimum. This therapy was continued for 42 days. No other immunosuppressive therapy was administered.

2.4. Measurement of White Blood Cell (WBC) Counts. Complete WBC counts on pig blood were carried out using automated methodology at the University of Pittsburgh Medical Center Central Laboratory, Presbyterian Hospital, Pittsburgh, PA.

2.5. Histopathologic Examination. After euthanasia of the pigs, necropsies were performed through a midline abdominal incision. Kidney specimens were taken at necropsy, placed in formalin, stained with hematoxylin and eosin, and examined microscopically. Kidney allograft rejection (acute cellular rejection) was scored by standard pathologic criteria according to the cooperative clinical trials in transplantation (CCTT) classification system and incorporated into the revised Banff criteria $[11,12]$.

2.6. Isolation and Storage of Peripheral Blood Mononuclear Cells (PBMC). PBMC from donor, recipient, and third-party pigs were isolated from heparinized blood by centrifugation over Ficoll-Paque PLUS (Amersham Biosciences, Piscataway, NJ), as described previously [13]. The total mononuclear cell fraction was washed twice with PBS (Gibco, Grand Island, NY) and then resuspended in FACS buffer (PBS containing $1 \%$ BSA and $0.1 \% \mathrm{NaN}_{3}$ ) for flow cytometry or in mixed lymphocyte reaction (MLR) medium (including 10\% controlled processed serum replacement type 3 [CPSR-3], Sigma, St Louis, MO) for MLR assay.

Donor and third-party pig PBMCs were stored in 10\% DMSO RPMI with $20 \%$ FBS and kept at $-80^{\circ} \mathrm{C}$ for $24 \mathrm{~h}$ before being stored in liquid nitrogen. For MLR, the cryopreserved donor and third-party pig PBMCs were thawed by a rapid rewarming procedure in a water bath at $37^{\circ} \mathrm{C}$ and washed with $10 \%$ FBS RPMI medium. 
2.7. Flow Cytometry. Fresh heparinized pig blood $(400 \mu \mathrm{L})$ was lysed $\times 2$ using ACK lysing solution to obtain pig WBCs, which were washed with PBS, resuspended with FACS buffer, and aliquoted into FACS tubes $(80 \mu \mathrm{L} /$ tube). Detection of $\mathrm{T}, \mathrm{B}, \mathrm{CD}^{+}$, and $\mathrm{CD} 8^{+}$cells was determined by incubating the pig WBC with, respectively, $10 \mu \mathrm{L}$ mouse anti-pig $\mathrm{CD} 3 \varepsilon$ FITC $\mathrm{mAb}$ at a dilution of $1: 64$, mouse anti-human CD21$\mathrm{PE} \mathrm{mAb}$ at $1: 16$, mouse anti-pig CD4a-PE mAb at $1: 16$, or mouse anti-pig CD8a-PE mAb at 1:64. (All mAbs were obtained from BD Biosciences Pharmingen, Franklin Lakes, $\mathrm{NJ})$. The samples were washed twice, and the cells resuspended with $200 \mu \mathrm{L}$ of FACS buffer. Data acquisition was performed with FACScan (Becton Dickinson, Mountain View, CA) using Cell Quest software. Dead cells were excluded from the analysis by side-scatter and propidium iodide staining ( $10 \mu \mathrm{L}$ added to each sample prior to FACS analysis). Data analysis was carried out using WinMDI software.

2.8. Mixed Lymphocyte Reaction (MLR). PBMC were isolated from fresh heparinized pig blood, as described above, to a concentration of $4 \times 10^{6}$ cells $/ \mathrm{mL}$. Pig PBMCs $(0.4 \times$ $10^{6}$ cells/well) were incubated with an equal number of irradiated stimulator cells $\left(0.4 \times 10^{6}\right.$ cells/well $)$ in $10 \%$ CPSR-3 RPMI medium at $37^{\circ} \mathrm{C}$ and $5 \% \mathrm{CO}_{2}$ using 96-well roundbottom plates (Corning, New York, NY) [13]. Stimulator cells were irradiated with $2,500 \mathrm{cGy}$ in a cesium ${ }^{137}$ irradiator. Each assay was performed in triplicate. After 4 days, $10 \mu \mathrm{L}$ of $3 \mathrm{H}$-thymidine-labeling medium $(1 \mu \mathrm{Ci} /$ well; New England Nuclear, Boston, MA) was added to each well. Cells were harvested after $18 \mathrm{~h}$ on glass-fiber filter mats with a cell harvester and were analyzed by beta counting on a liquid scintillation counter. Responder pig PBMCs were stimulated with autologous, donor, and third-party pig PBMC. The results were recorded as stimulation index (ratio of the response compared to that of autologous cells).

\section{Experimental Groups}

3.1. Study 1: Experiments to Determine Optimum Dose of Irradiation to Deplete Leukocytes without Damaging the Kidneys. An attempt was made to determine (i) the optimum dose of irradiation that would deplete leukocytes without damaging the kidneys, and (ii) the optimum period that should elapse before the kidneys should be excised for Tx. Pigs received either low-dose $(700+800 \mathrm{cGy} ; n=3)$ or high-dose $(700$ $+1300 \mathrm{cGy}, n=3$ ) irradiation. All pigs were euthanized 60 or 72 hours after irradiation for examination of the kidneys.

\subsection{Study 2: Experiments to Determine the Effect of Donor Pre-Transplant Total Body Irradiation on Kidney Allograft Sur- vival. Life-supporting kidney Tx was carried out in the fol- lowing experiments.}

Experiment 1. A kidney from a nonirradiated donor into a recipient im-munosuppressed with tacrolimus for 42 days.

Experiment 2. A kidney from a nonirradiated donor into a nonimmunosuppressed recipient.
Experiment 3. A kidney from an irradiated donor $(700+$ $1300 \mathrm{cGy}$ carried out $72 \mathrm{~h}$ previously) into a nonimmunosuppressed recipient.

In Experiments 2 and 3, the donor and recipient pairs were reported not to be siblings.

\section{Results}

4.1. Study 1: Experiments to Determine Optimum Dose of Total Body Irradiation to Deplete Leukocytes without Damaging the Kidneys. During the 60-72 h after irradiation and before euthanasia, no pig experienced any vomiting or other obvious side effects from the irradiation, and no hematuria was observed. The cell counts in the blood of all pigs receiving either low-dose $(n=3)$ or high-dose $(n=3)$ irradiation indicated that the number of WBC, T cells, and B cells decreased significantly within $24 \mathrm{~h}$ of irradiation (Figure 1 ). The $\mathrm{CD}^{+}, \mathrm{CD}^{+}, \mathrm{CD}^{+}$, and $\mathrm{B}$ cell counts in the blood were all $<100 / \mathrm{mm}^{3} 24 \mathrm{~h}$ after irradiation. By $72 \mathrm{~h}$, there were almost no cells in the blood, and the occasional cells that remained were polymorphonuclear neutrophils.

The histopathologic examination of the kidneys of pigs that had received low-dose irradiation showed occasional residual passenger leukocytes, with congestion of blood vessels, tubular vacuolization, and some tubular epithelial apoptosis (Figure 2). The kidneys of pigs that had received highdose irradiation showed very rare leukocytes, apoptotic cells, and minor hemorrhages in the medulla (Figure 3).

We concluded that low-dose irradiation depleted most, but not all, leukocytes in the kidneys. High-dose irradiation caused virtually complete depletion of leukocytes within $72 \mathrm{~h}$ and more apoptosis of cells, but also slightly more congestion and hemorrhage in the kidneys (Figure 3(d)), suggesting some radiation injury, possibly related to inadequate protection of the kidneys during the procedure. The period that had elapsed between irradiation and microscopic examination of the kidneys (60 h or $72 \mathrm{~h}$ ) did not appear to influence the histopathologic features.

As there was a minimal number of leukocytes remaining in the blood of the donor $72 \mathrm{~h}$ after high-dose irradiation, and as we hypothesized that any remaining cells might be dying, we determined to test whether the donor treatment had had any effect on kidney survival after Tx. For this study, the higher dose of irradiation was selected, that is, $700 \mathrm{cGy}$ and a further $1300 \mathrm{cGy}$ with both kidneys shielded, which was carried out very carefully to avoid injury to the kidneys. Seventy-two hours was selected as the period that would elapse before kidney excision and Tx.

\subsection{Study 2: Experiments to Determine the Effect of Donor Pre- Transplant Total Body Irradiation on Kidney Allograft Sur- vival}

4.2.1. Life-Supporting Kidney Tx from a Nonirradiated Donor into an Immunosuppressed Recipient. This experiment was carried out to document that the surgical technique was successful. To allow differentiation between graft failure from 

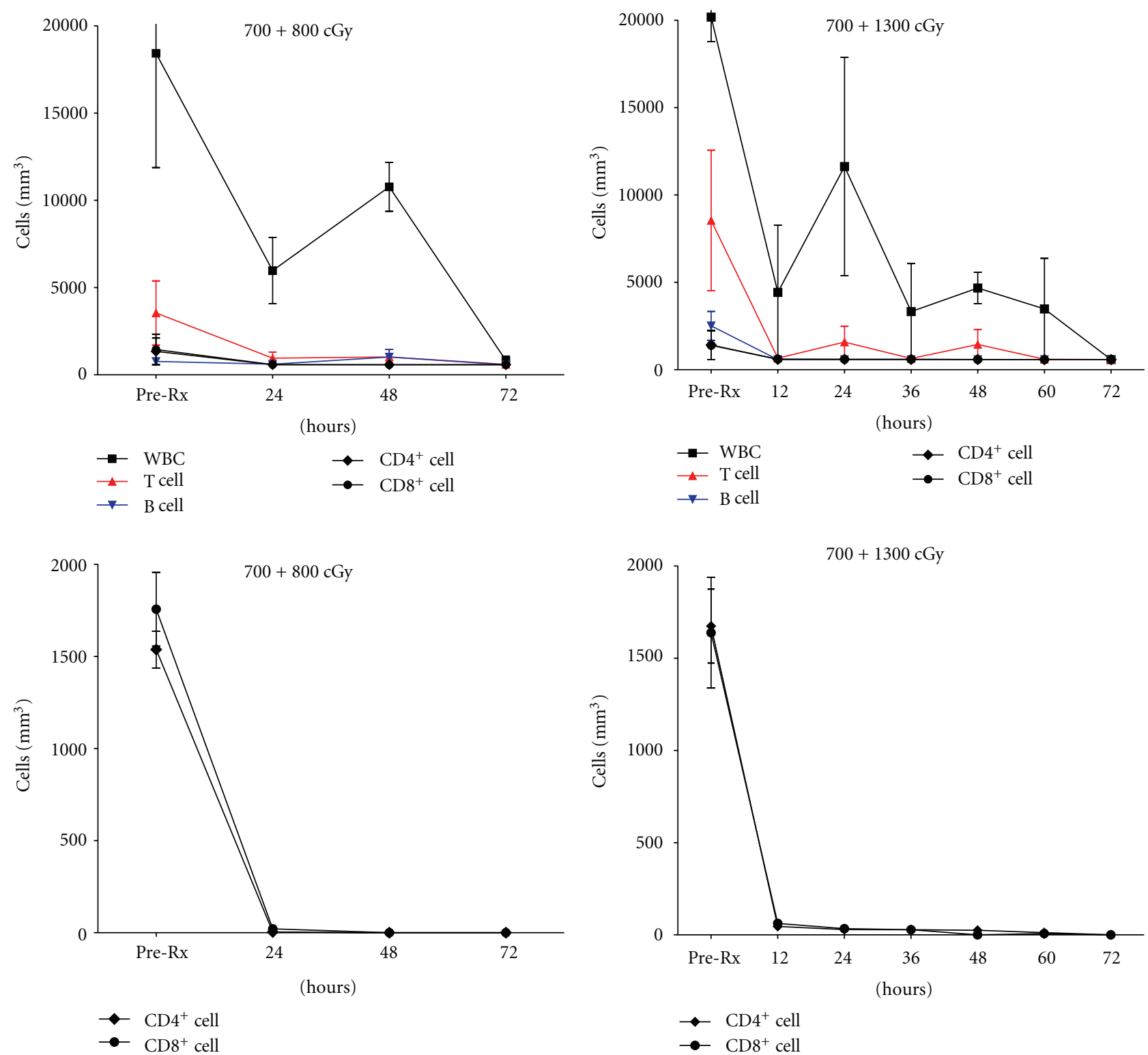

(a)

(b)

Figure 1: Cell counts in the blood of pigs receiving (a) low-dose or (b) high-dose irradiation. (a) Mean total WBC, T and B cell counts (left upper), and $\mathrm{CD}^{+}$and $\mathrm{CD}^{+}$cell counts (left lower) of 3 pigs receiving WBI of $700 \mathrm{cGy}$ and a further $800 \mathrm{cGy}$ with both kidneys shielded. (b) Mean total WBC, T and B cell counts (right upper), and CD4 ${ }^{+}$and $\mathrm{CD} 8^{+}$cell counts (right lower) of 3 pigs receiving WBI of $700 \mathrm{cGy}$ and a further $1300 \mathrm{cGy}$ with both kidneys shielded. Although T and B cell counts decreased rapidly to $<100$ cells/mm $\mathrm{mm}^{3}$ within $24 \mathrm{~h}$ after irradiation, the total WBC count did not reach such a low number for $72 \mathrm{~h}$. The remaining WBCs were largely polymorphonuclear neutrophils.

surgical complication and rejection, tacrolimus was administered for 42 days to prevent rejection in the early postTx course. The kidney clearly remained viable throughout the period of followup since the serum creatinine was maintained $<176.8 \mu \mathrm{mol} / \mathrm{L}(<2 \mathrm{mg} / \mathrm{dL})$ for almost 5 months after discontinuation of tacrolimus immunosuppression (Figure 4). The pig was euthanized electively 188 days after kidney Tx. At all time intervals (both before and after Tx), the MLR showed a very low stimulation index to donorspecific stimulators (not shown). At necropsy, histological examination of the transplanted kidney showed minimal abnormality.

\subsubsection{Life-Supporting Kidney Tx from a Nonirradiated Donor} into a Nonimmunosuppressed Recipient. The serum creatinine increased to $>884 \mu \mathrm{mol} / \mathrm{L}(>10 \mathrm{mg} / \mathrm{dL})$ within 7 days (Figure 5). MLR showed a moderately high response before Tx (stimulation index 18) with a reduced response at the time of rejection (stimulation index 7) (not shown). 


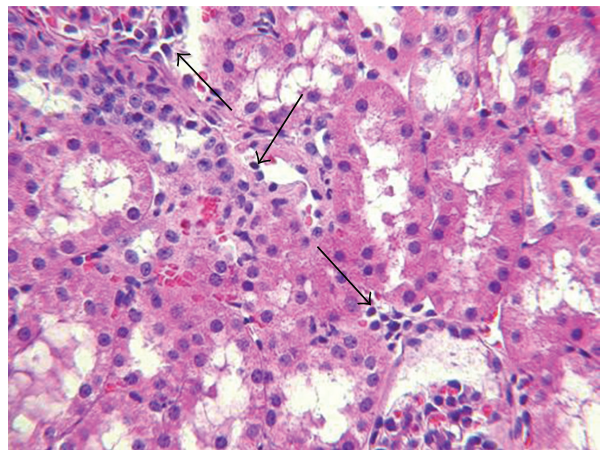

(a)

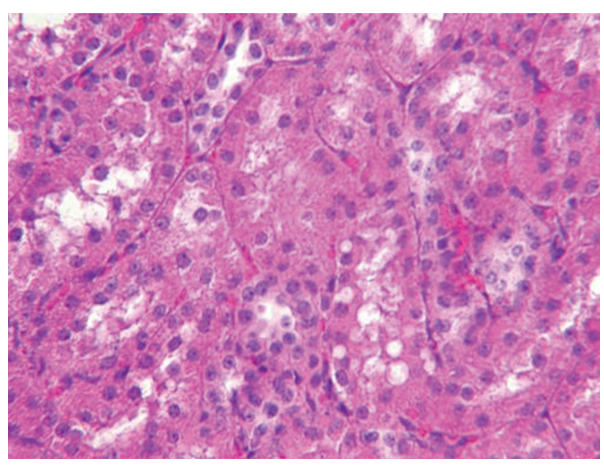

(c)

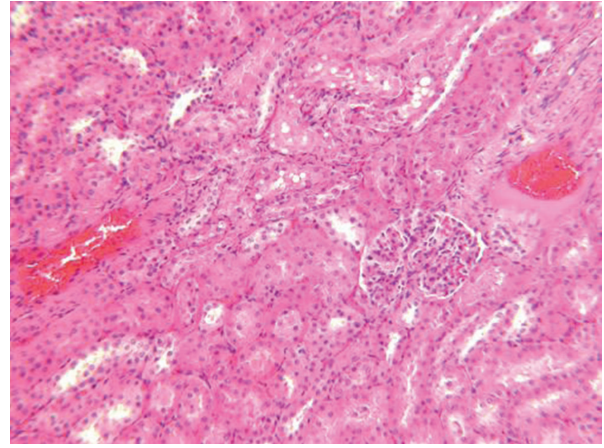

(b)

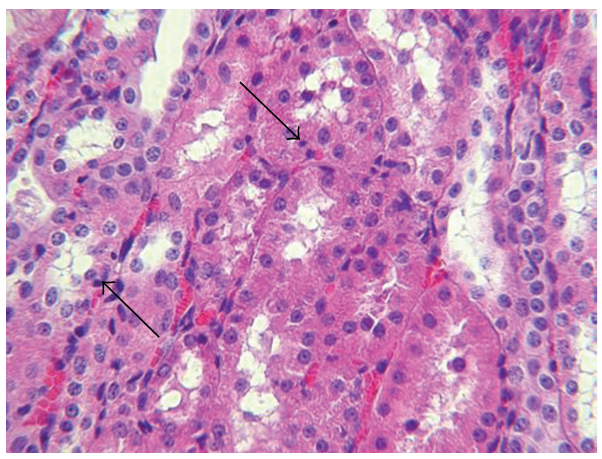

(d)

FIgURE 2: Histopathology of kidneys from a pig that received WBI $72 \mathrm{~h}$ previously of $700 \mathrm{cGy}$ and a further $800 \mathrm{cGy}$ with both kidneys shielded (H\&E, magnification $\times 100)$. (a) A few passenger leukocytes can be detected in the interstitium (arrows). (b) Congestion is present in some peritubular blood vessels. (c) Tubular vacuolization and cloudy swelling of tubular cells are present. (d) Tubular epithelial apoptosis can be detected (arrows).

Histopathology showed the typical features of acute cellular rejection (not shown).

\subsubsection{Life-Supporting Kidney Tx from an Irradiated Donor} into a Nonimmunosuppressed Recipient. Following irradiation, the data were consistent with those observed in the preliminary irradiation study (Figure 1). By the day of kidney Tx (day 0-72 $\mathrm{h}$ after TBI), there were virtually no $\mathrm{T}, \mathrm{B}, \mathrm{CD} 4^{+}$, or $\mathrm{CD}^{+}$cells remaining in the donor blood. After $\mathrm{Tx}$, however, the serum creatinine increased to $>884 \mu \mathrm{mol} / \mathrm{L}(>10 \mathrm{mg} / \mathrm{dL})$ within 9 days (Figure 5). Both before irradiation and at the time of graft failure, the MLR showed a very low response to donor-specific PBMC (not shown, suggesting significant inbreeding in some members of the herd). Histopathology of the graft showed the typical features of acute cellular rejection (not shown). No hematuria was observed in the donor pig after irradiation or in the recipient after kidney Tx.

\section{Discussion}

To determine the dose of irradiation required to deplete all passenger leukocytes, rodent models will not provide the data required since the dose of irradiation likely to achieve the desired effect will be different from that in large animals. It is known that unfractionated doses of irradiation have a greater effect than fractionated doses in destroying all of the passenger leukocytes [14, 15]. In humans, a "standard" 10 Gy single dose of TBI appears to be consistently more toxic than fractionated TBI regimens delivering from 12 to $15 \mathrm{~Gy}[14,15]$. It is also known that animals will survive for approximately 14 days before succumbing from the effects of a lethal dose of irradiation [16]. Mezrich et al. [6]. found that some pigs did not tolerate 1,500 cGy of TBI and developed renal failure secondary to irradiation toxicity; a dose of 1,000 cGy was well tolerated.

The irradiated pigs in the present study were euthanized 60-72 h after irradiation to prevent irradiation-related complications. All cell types measured in the blood (WBC, T, $\left.\mathrm{CD}^{+}, \mathrm{CD}^{+}, \mathrm{B}\right)$ were significantly depleted by both dosages of irradiation tested and particularly by the higher dose (700 + 1300 cGy). By $72 \mathrm{~h}$ after TBI, T and B lymphocyte depletion from the blood was total except for an occasional polymorphonuclear leukocyte. The dose of irradiation selected may not have depleted all $\mathrm{T}$ and $\mathrm{B}$ cells in the thymus and/or spleen and/or bone marrow; one of the limitations of our study is that we did not monitor for leukocytes in these organs. At the higher dose of irradiation, minor injury to the kidneys was seen on histological examination, possibly related to inadequate protection of the kidneys. The pathophysiology of radiation injury of the kidney is poorly understood, and its presentation can be acute and 


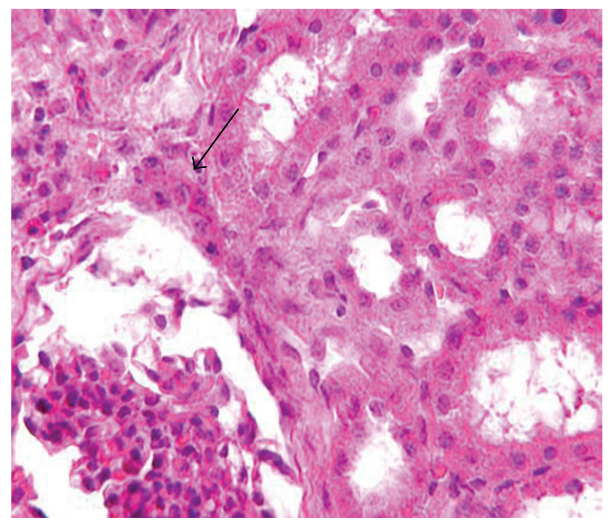

(a)

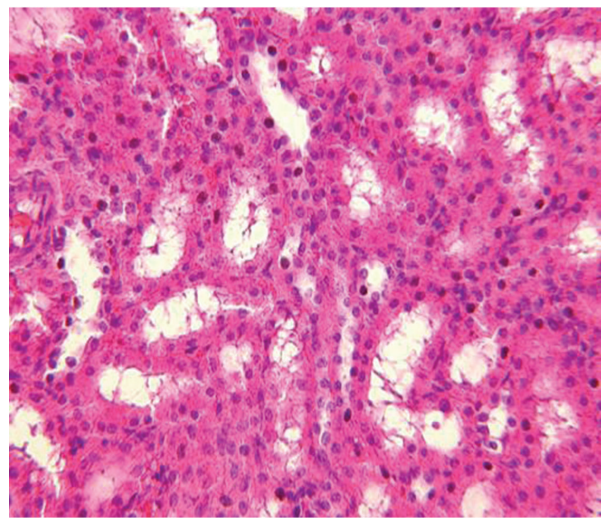

(c)

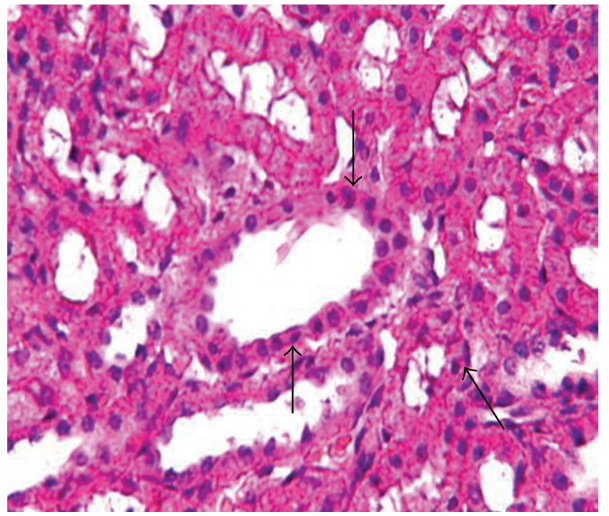

(b)

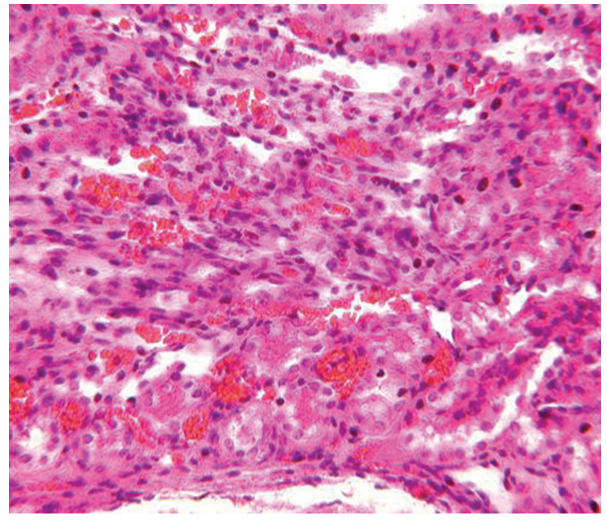

(d)

FIGURE 3: Histopathology of a kidney from a pig that received WBI $72 \mathrm{~h}$ previously of $700 \mathrm{cG}$ and a further $1300 \mathrm{cGy}$ with both kidneys shielded (H\&E, magnification $\times 100)$. (a) A few passenger leukocytes can be detected in the interstitium (arrow). (b) A few apoptotic cells can be detected (arrows). (c) Minor hemorrhages are seen in the interstitium of medulla. (d) Congestion of peritubular blood vessels and interstitial edema can be seen.

irreversible or subtle, with a gradual progressive dysfunction over years [17].

We would conclude that perhaps neither of the irradiation regimens used was totally successful in depleting all passenger leukocytes in the donor kidney. Higher doses of irradiation, however, might have been associated with increased morbidity in the donor pig, as reported by Mezrich et al. [6] (and were, in fact, opposed on humane grounds by the Institutional Animal Care and Use Committee). We were, therefore, not able to test any higher doses of irradiation in this study. Nevertheless, despite the possibility that depletion might have been incomplete, we decided to test the effect of the donor irradiation by kidney Tx.

A control kidney $\mathrm{Tx}$ in a recipient that received a 42 day course of tacrolimus in the absence of irradiation to the donor indicated that the kidney transplant procedure was technically successful and that, when a kidney was transplanted from an outbred pig from the same herd, operational tolerance developed. This result suggested that either (i) the donor and recipient pigs were closely related, for example, siblings, or (ii) the herd was sufficiently inbred to allow kidney $\mathrm{Tx}$ in the absence of graft rejection, particularly in the presence of a 42-day course of tacrolimus.
The pig is known to be a relatively easy Tx model in which to develop tolerance to a kidney allograft if a short course of immunosuppressive therapy is administered. Kidney allografts between the Massachusetts General Hospital (NIH) [18] MHC two-haplotype class I mismatched miniature swine that receive a 12-day course of cyclosporine are uniformly accepted [19]. Using the same regimen, a renal allograft across a MHC two-haplotype full-mismatch barrier is rejected, but tolerance can be achieved if highdose tacrolimus replaces cyclosporine [20]. In the present study (in which the MHC profiles of the donor-recipient pairs were not investigated) the long-term graft survival in Experiment 1 indicates that operational tolerance was achieved by a 42-day course of tacrolimus at standard dosage.

When no immunosuppressive therapy was administered to pigs that received a kidney from either a nonirradiated donor or irradiated donor, acute cellular rejection developed within 7-9 days. In both cases, the donor and recipient pigs were not siblings (but the MHC profile was not known, and the MLR was weak between one donor-recipient pair). This indicated that irradiation of the donor (in the dosage administered) did not prevent rejection (possibly because depletion of donor passenger leukocytes had been 


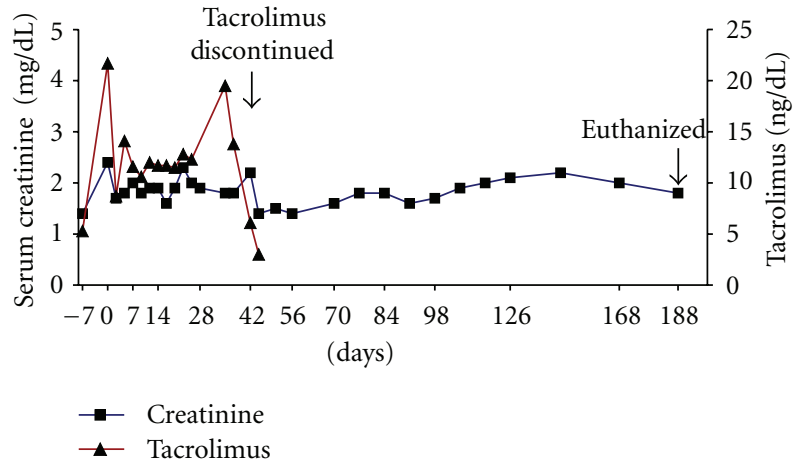

FIGURE 4: Life-supporting kidney Tx was carried out from a nonirradiated donor into a recipient immunosuppressed for 42 days with tacrolimus. Efforts were made to maintain the $12 \mathrm{~h}$ blood trough level between $10-15 \mathrm{ng} / \mathrm{mL}$. Serum creatinine levels remained between $132.6-176.8 \mu \mathrm{mol} / \mathrm{L}(1.5-2.0 \mathrm{mg} / \mathrm{dL})$ for almost 5 months after discontinuing tacrolimus. The pig was euthanized electively 188 days after kidney $T x$.

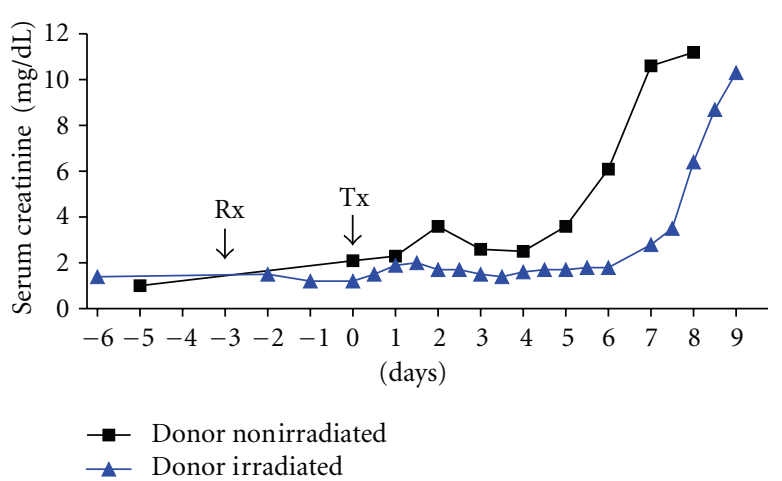

FIGURE 5: Life-supporting kidney Tx was carried out from (i) a nonirradiated donor (squares) and (ii) an irradiated donor (triangles) into nonsibling recipients without immunosuppressive therapy. The serum creatinine levels of the pigs rose to $>884 \mu \mathrm{mol} / \mathrm{L}$ $(>10 \mathrm{mg} / \mathrm{dL})$ within 7 and 9 days, respectively. Rx: day of irradiation to donor (day -3$)$; Tx: day of kidney Tx (day 0$)$.

incomplete or because antigens from dead leukocytes were presented by recipient antigen-presenting cells). Furthermore, in the absence of a course of tacrolimus, operational tolerance was not achieved after kidney Tx between members of this herd.

The present study also suggests that MLR data in pigs may not be indicative of graft outcome, as illustrated by Experiment 2 (in which the pre-Tx response on MLR was high) and Experiment 3 (in which it was very low), and yet graft failure from acute cellular rejection developed in similar time frames (although TBI to the donor may have influenced the outcome).

In view of the difficulty in completely depleting passenger leukocytes in the donor and the lack of any obvious difference in survival between the two kidney allografts, we did not feel that there was any purpose in pursuing the project further.
It would be possible to excise the kidney from the donor pig and irradiate it ex vivo. However, there might be several problems with this approach. Lymphocyte numbers significantly decrease after irradiation of pigs in vivo or of pig blood in vitro in a dose-dependent manner, but reduction of lymphocyte numbers after irradiation in vivo proceeds much faster than after irradiation in vitro [21]. Furthermore, a significantly different response to irradiation is achieved in vivo than in vitro or ex vivo. To have an optimum effect, irradiation depends on high oxygen content in the tissues $[22,23]$. There is likely to be a low oxygen partial pressure in a cold ex vivo kidney, and this would reduce the effect of irradiation on the passenger leukocytes within the kidney. In view of a lack of background and experience in this approach, it would probably require several experiments to elucidate the optimum dosage if the organ was to be subjected to irradiation ex vivo.

Combining irradiation of the donor with the administration of a cytotoxic agent, for example, cyclophosphamide (although our evidence is that this drug is ineffective in pigs [24]) might result in more complete depletion of leukocytes. In the present study, monitoring leukocyte depletion in the thymus, spleen, or bone marrow would have provided a more accurate picture of the number and phenotype of the leukocytes remaining viable.

In summary, TBI at the doses administered was well tolerated by the pigs, but was not completely successful in depleting all passenger leukocytes, and this may not have completely prevented a direct T-cell response. Furthermore, we could not exclude presentation of donor antigens from dead or dying passenger leukocytes transplanted with the graft by recipient antigen-presenting cells, and therefore the indirect response may have been active.

Although kidney $\mathrm{Tx}$ was carried out in only one pig that had received a kidney from an irradiated donor, graft survival was only minimally prolonged compared with that of a nonirradiated kidney. We conclude that the irradiation regimens used in the present study were insufficient to deplete all leukocytes and did not prolong kidney graft survival after Tx. Further investigation of means of effectively depleting passenger leukocytes in large animals is required. The study did not rule out that TBI of the donor may be detrimental to kidney graft survival, though the results do not support this conclusion.

However, one other factor has to be considered in assessing the results. Porcine endothelial cells express MHC class II molecules constitutively, at least in some vascular beds [25, $26]$, indicating that they will be recognized by $\mathrm{CD} 4^{+} \mathrm{T}$ cells. They also constitutively express porcine B7 molecules [27], indicating that they may activate $\mathrm{CD} 4^{+}$cells just as efficiently as hematopoietic cells. It may be, therefore, that, even in the complete absence of passenger leukocytes in a transplanted pig organ allograft, acute rejection may still develop unless immunosuppressive therapy is administered for a period of time.

If pigs are to be used for these studies, it would be essential to have donors and recipients known to be of different MHC, and some immunosuppressive therapy would appear to be required, though the intensity and duration of the 
therapy would require careful investigation, making the model a difficult one. As it is so relatively easy to induce tolerance in a pig (in contrast, e.g. to a nonhuman primate or human) it may be that the pig is not the ideal animal model in which to explore this phenomenon.

\section{Abbreviations}

MLR: Mixed lymphocyte reaction

PBMCs: Peripheral blood mononuclear cells

TBI: Total body irradiation

Tx: Transplantation

WBC: White blood cells.

\section{Conflict of Interests}

The authors declare there is no conflict of interest.

\section{Acknowledgments}

H.-C. Tai, is a recipient of a grant from National Taiwan University Hospital, Taiwan. These studies were supported in part by the Eberly Fund for Transplantation at the Thomas E. Starzl Transplantation Institute of the University of Pittsburgh.

\section{References}

[1] C. P. Larsen, J. M. Austyn, and P. J. Morris, "The role of graft-derived dendritic leukocytes in the rejection of vascularized organ allografts: recent findings on the migration and function of dendritic leukocytes after transplantation," Annals of Surgery, vol. 212, no. 3, pp. 308-317, 1990.

[2] A. J. Demetris, N. Murase, A. S. Rao, J. J. Fung, and T. E. Starzl, "The dichotomous functions of passenger leukocytes in solid-organ transplantation," Advances in Nephrology from the Necker Hospital, vol. 24, pp. 341-354, 1995.

[3] K. J. Wood, "Passenger leukocytes and microchimerism: what role in tolerance induction?" Transplantation, vol. 75, supplement 9, pp. 17S-20S, 2003.

[4] S. Oluwole, M. A. Hardy, and T. Wang, "Donor pretreatment: rat heart allograft survival and measurement of passenger leukocyte depletion with indium-111," Transplantation, vol. 30, no. 1, pp. 31-33, 1980.

[5] N. Murase, Q. Ye, M. A. Nalesnik et al., "Immunomodulation for intestinal, transplantation by allograft irradiation, adjunct donor bone marrow infusion, or both," Transplantation, vol. 70, no. 11, pp. 1632-1641, 2000.

[6] J. D. Mezrich, K. Yamada, R. S. Lee et al., "Induction of tolerance to heart transplants by simultaneous cotransplantation of donor kidneys may depend on a radiation-sensitive renalcell population," Transplantation, vol. 76, no. 4, pp. 625-631, 2003.

[7] R. J. Steptoe, R. K. Patel, V. M. Subbotin, and A. W. Thomson, "Comparative analysis of dendritic cell density and total number in commonly transplanted organs: morphometric estimation in normal mice," Transplant Immunology, vol. 8, no. 1, pp. 49-56, 2000.

[8] L. R. Pennington, K. Sakamoto, F. A. Popitz-Bergez et al., "Bone marrow transplantation in miniature swine. I. Development of the model," Transplantation, vol. 45, no. 1, pp. 21-26, 1988.
[9] F. A. Popitz-Bergez, K. Sakamoto, L. R. Pennington et al., "Bone marrow transplantation in miniature swine. II. Effect of selective genetic differences on marrow engraftment and recipient survival," Transplantation, vol. 45, no. 1, pp. 27-31, 1988.

[10] C. V. Smith, T. Suzuki, P. C. Guzzetta et al., "Bone marrow transplantation in miniature swine: IV. Development of myeloablative regimens that allow engraftment across major histocompatibility barriers," Transplantation, vol. 56, no. 3, pp. 541-549, 1993.

[11] L. C. Racusen, K. Solez, R. B. Colvin et al., "The Banff 97 working classification of renal allograft pathology," Kidney International, vol. 55, no. 2, pp. 713-723, 1999.

[12] R. B. Colvin, A. H. Cohen, C. Saiontz et al., "Evaluation of pathologic criteria for acute renal allograft rejection: reproducibility, sensitivity, and clinical correlation," Journal of the American Society of Nephrology, vol. 8, no. 12, pp. 19301941, 1997.

[13] H.-C. Tai, X. Zhu, H. Hara et al., "The pig-to-primate immune response: relevance for xenotransplantation," Xenotransplantation, vol. 14, no. 3, pp. 227-236, 2007.

[14] J. M. Cosset, T. Girinsky, E. Malaise, M. P. Chaillet, and J. Dutreix, "Clinical basis for TBI fractionation," Radiotherapy and Oncology, vol. 18, no. 3331, pp. 60-67, 1990.

[15] J. M. Cosset, G. Socie, B. Dubray, T. Girinsky, A. Fourquet, and E. Gluckman, "Single dose versus fractionated total body irradiation before bone marrow transplantation: radiobiological and clinical considerations," International Journal of Radiation Oncology Biology Physics, vol. 30, no. 2, pp. 477-492, 1994.

[16] J. R. Rust, B. F. Trum, J. L. Wilding, C. S. Simons, and C. L. Comar, "Lethal dose studies with burros and swine exposed to whole body cobalt-60 irradiation," Radiology, vol. 62, pp. 569-574, 1954.

[17] L. A. Dawson, B. D. Kavanagh, A. C. Paulino et al., "Radiationassociated kidney injury," International Journal of Radiation Oncology Biology Physics, vol. 76, supplement 3, pp. S108S115, 2010.

[18] D. H. Sachs, "MHC homozygous miniature swine," in Swine as Models in Biomedical Research, M. M. Swindle, D. C. Moody, and L. D. Phillips, Eds., pp. 3-15, Iowa State University Press, Ames, Iowa, USA, 1992.

[19] B. R. Rosengard, C. A. Ojikutu, P. C. Guzzetta et al., "Induction of specific tolerance to class I-disparate renal allografts in miniature swine with cyclosporine," Transplantation, vol. 54, no. 3, pp. 490-497, 1992.

[20] R. Utsugi, R. N. Barth, R. S. Lee et al., "Induction of transplantation tolerance with a short course of tacrolimus (FK506): i. Rapid and stable tolerance to two-haplotype fully MHCmismatched kidney allografts in miniature swine," Transplantation, vol. 71, no. 10, pp. 1368-1379, 2001.

[21] L. Zarybnicka, Z. Sinkorova, J. Sinkora et al., "Sensitivity of porcine peripheral blood leukocytes to gamma irradiation in vivo, in vitro and ex vivo," International Journal of Radiation Biology, vol. 87, no. 5, pp. 491-498, 2011.

[22] E. J. Hall, M. Astor, J. Bedford et al., "Basic radiobiology," American Journal of Clinical Oncology, vol. 11, no. 3, pp. 220252, 1988.

[23] M. E. Robbins, W. Zhao, C. S. Davis, S. Toyokuni, and S. M. Bonsib, "Radiation-induced kidney injury: a role for chronic oxidative stress?" Micron, vol. 33, no. 2, pp. 133-141, 2002.

[24] G. Echeverri and D. K. C. Cooper, "Ineffectiveness of cyclophosphamide at clinical dosages in pigs," Annals of Transplantation, vol. 14, no. 4, pp. 91-92, 2009. 
[25] C. A. Bravery, P. Batten, M. H. Yacoub, and M. L. Rose, "Direct recognition of SLA- and HLA-like class II antigens on porcine endothelium by human $\mathrm{T}$ cells results in $\mathrm{T}$ cell activation and release of interleukin-2," Transplantation, vol. 60, no. 9, pp. 1024-1033, 1995.

[26] J. K. Choo, J. D. Seebach, V. Nickeleit et al., "Species differences in the expression of major histocompatibility complex class II antigens on coronary artery endothelium: implications for cell-mediated xenoreactivity," Transplantation, vol. 64, no. 9, pp. 1315-1322, 1997.

[27] T. A. Davis, N. Craighead, A. J. Williams, A. Scadron, C. H. June, and K. P. Lee, "Primary porcine endothelial cells express membrane-bound B7-2 (CD86) and a soluble factor that co-stimulate cyclosporin A-resistant and CD28-dependent human T cell proliferation," International Immunology, vol. 8, no. 7, pp. 1099-1111, 1996. 


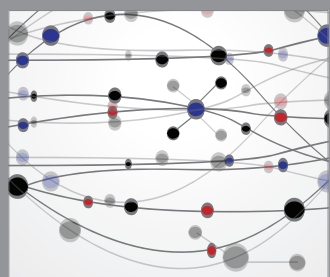

The Scientific World Journal
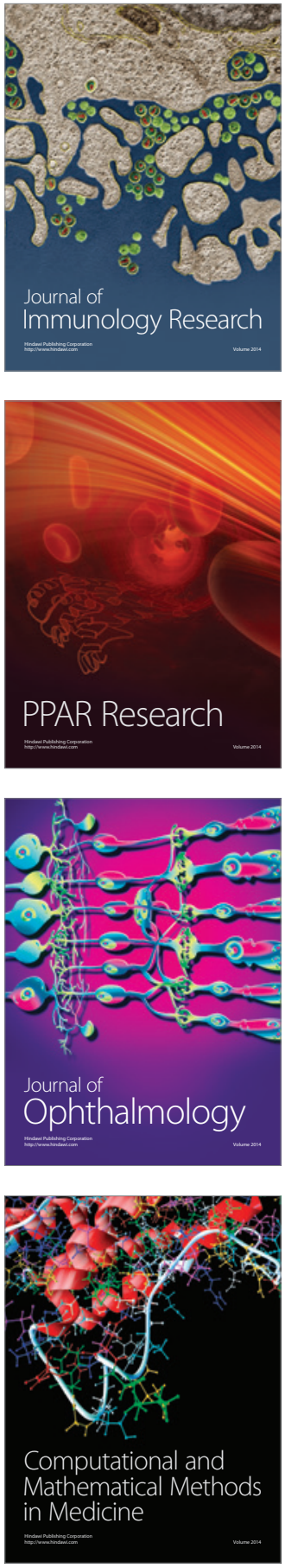

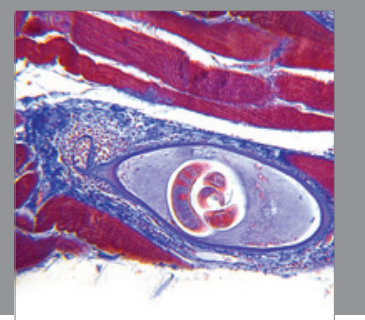

Gastroenterology

Research and Practice
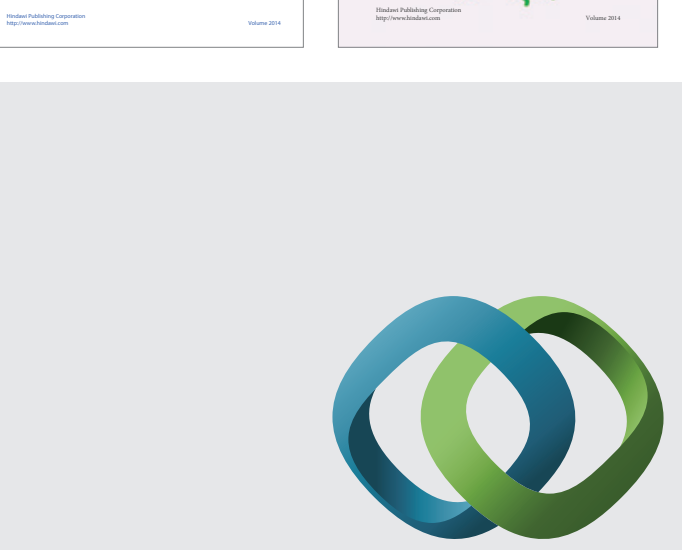

\section{Hindawi}

Submit your manuscripts at

http://www.hindawi.com
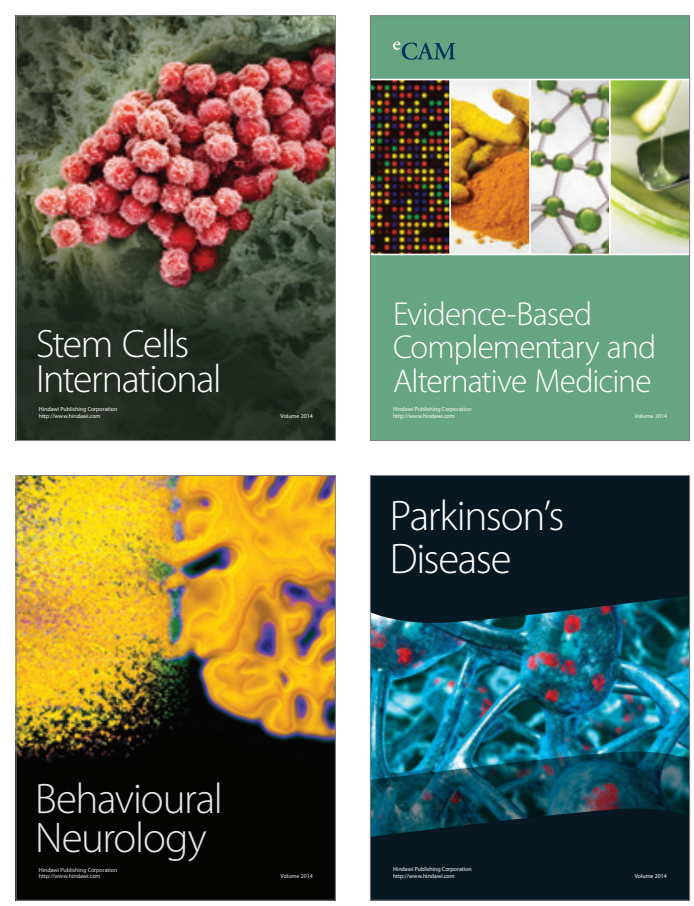

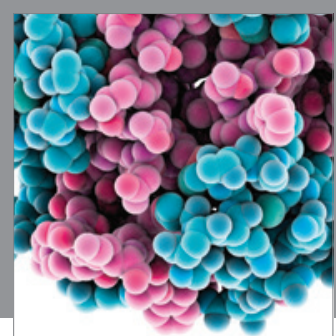

Journal of
Diabetes Research

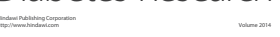

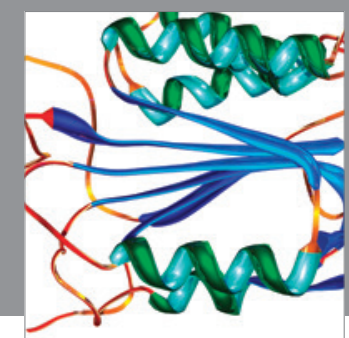

Disease Markers
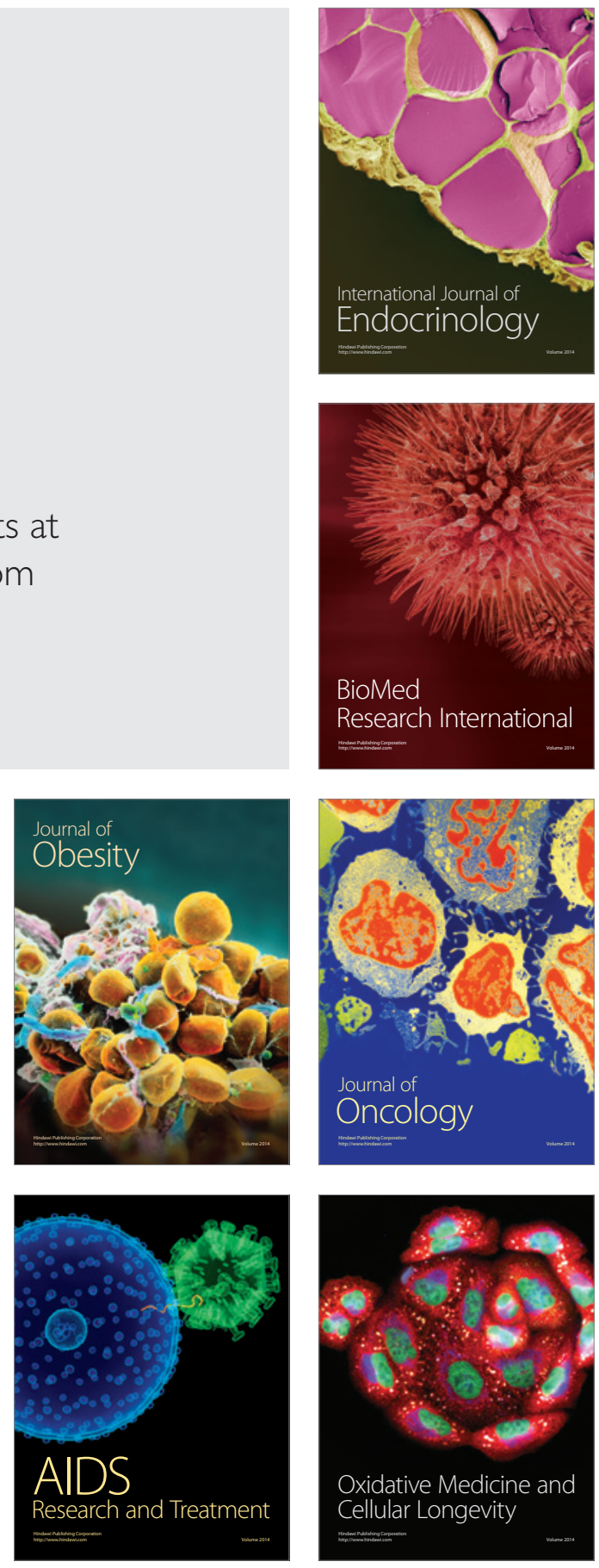